\title{
Shell model for quasi-two-dimensional turbulence
}

\author{
G. Boffetta, ${ }^{1}$ F. De Lillo, ${ }^{1}$ and S. Musacchio ${ }^{2}$ \\ ${ }^{1}$ Dipartimento di Fisica Generale and INFN, \\ Università di Torino, via P.Giuria 1, I-10125 Torino, Italy \\ ${ }^{2}$ CNRS, Lab. J.A. Dieudonné UMR 6621, \\ Parc Valrose, F-06108 Nice, France
}

(Received 3 March 2011; published 3 June 2011)

\begin{abstract}
We discuss the possibility to introduce geometrical constraints in shell models of turbulence in order to mimic the turbulent dynamics that takes place in fluid layers with large aspect ratio. By using a scale-dependent set of coupling parameters, we are able to resolve both scales larger and smaller than a geometrical dimension of the flow. The proposed model is able to resolve with high accuracy the split energy cascade phenomenon recently observed in such flows, and allows us to investigate in detail the scaling properties of turbulent convection confined in narrow convective cells.
\end{abstract}

DOI: 10.1103/PhysRevE.83.066302

PACS number(s): 47.27.E-

The dynamics of fluid layers whose depth is much smaller than their extension attracts a broad attention within the research in fluid mechanics, not only because of its fundamental relevance in geophysical applications, but also because of the scientific interest provoked by its peculiar behavior. The phenomenology of such flows is somehow puzzling. At horizontal scale much larger than the depth $h$ of the fluid layer it exhibits specific features of idealized two-dimensional (2D) flows, in spite of the fact that at small scales the flow can have a genuine three-dimensional (3D) turbulent behavior [1,2].

Investigating these properties by means of direct numerical simulations of Navier-Stokes equations can be very challenging, because it requires resolving both the $3 \mathrm{D}$ turbulent flow at scales much smaller than $h$, up to the viscous scale $\eta$, and the 2D flow, which take place at scales much larger than $h$. The extension of the two inertial ranges which can be simultaneously achieved by exploiting actual computational resources is still rather limited.

An alternative approach with respect to direct numerical simulation can be provided by the shell models of turbulence. A large number of these models have been proposed in the past (see, e.g., [3-8]) in order to mimic the phenomenology of turbulent cascades by means of simplified equations for the complex variables $u_{n}$ (with $1 \leqslant n \leqslant N$ ), which are thought of as representatives of the Fourier components of the velocity field associated to the wave numbers $k_{n}$. The latter are chosen to be geometrically spaced as $k_{n}=k_{0} \lambda^{n}$, thus allowing us to achieve a broad inertial range even with a small number $N$ of variables. Here we show that a simple modification of standard shell models of Navier-Stokes and Boussinesq equations provides suitable models which reproduce many interesting features of the turbulent cascades of quasi-2D fluid layers.

Let us consider the shell model

$$
\frac{d u_{n}}{d t}=i \Phi_{n}(u, u)-D_{n} u_{n}+f_{n}
$$

where the nonlinear operator $\Phi_{n}(u, u)$, which models the interaction between shells, is chosen as in [7] $\Phi_{n}(u, u)=$ $a k_{n+1} u_{n+1}^{*} u_{n+2}+b k_{n} u_{n-1}^{*} u_{n+1}-c k_{n-1} u_{n-1} u_{n-2}$. The external force $f_{n}$ is active on the shell $n=n_{f}$ and the dissipative term has the form $D_{n}=\nu k_{n}^{2 p}+\mu k_{n}^{-2 q}$, where $\nu$ and $\mu$ are the viscosity and friction coefficients, respectively. This shell model has two inviscid conserved quantities:

$$
E=\frac{1}{2} \sum_{n=1}^{N}\left|u_{n}\right|^{2}, \quad H=\frac{1}{2} \sum_{n=1}^{N}\left(\frac{a}{c}\right)^{n}\left|u_{n}\right|^{2},
$$

provided that the coefficients $(a, b, c)$ satisfy the relation $a+$ $b+c=0$, and it is able to reproduce the phenomenology of $2 \mathrm{D}$ and $3 \mathrm{D}$ turbulent cascades, depending on the value of the ratio $c / a$. The 3D-like regime is obtained for $-1<c / a<0$. In this range of parameters the only positive definite inviscid invariant is the energy $E$, which is transferred by the interaction between shells toward large wave numbers, mimicking the direct energy cascade of $3 \mathrm{D}$ turbulence. In the range $0<c / a<$ 1 also the inviscid invariant $H$ is positive definite, and can be conveniently rewritten (up to a numerical constant $k_{0}^{\beta}$ ) as $H=$ $\frac{1}{2} \sum_{n=1}^{N} k_{n}^{\beta}\left|u_{n}\right|^{2}$ where $\beta=\ln (a / c) / \ln (\lambda)$. For $\lambda^{-2 / 3}<c / a<$ 1 it has been shown that the system displays a $2 \mathrm{D}$-like regime characterized by a double cascade of $E$ and $H$, with a negative flux of $E$ toward small wave numbers and a positive flux of $H$ toward large wave numbers [9]. It has to be noticed that in this range of parameters $H$ has not the physical dimension of enstrophy, being $0<\beta<2 / 3$. The value $c / a=\lambda^{-2}$, at which $H$ has the same dimension of enstrophy, is at the border between a regime of equipartition of $E$ and $H$ (for $0<c / a<$ $\lambda^{-2}$ ) and a regime of equipartition of $E$ and direct cascade of $H$ (for $\lambda^{-2}<c / a<\lambda^{-2 / 3}$ ) [9-11].

A suitable shell model for a fluid layer of depth $h$ should be able to mimic both the 3D behavior at high wave numbers $k_{n}>$ $k_{h} \sim 1 / h$ and the 2D phenomenology for $k_{n}<k_{h}$. Because shell models have no dimensionality, this can be obtained by allowing the coefficients $(a, b, c)$ to assume two different sets of values for a different range of shells. For the dynamics of shell variables $u_{n}$ with $n>n_{h}$ one sets the parameters to values corresponding to the $3 \mathrm{D}$-like behavior of the model, i.e., $-1<c / a<0$. Conversely the parameters for the shells $n<n_{h}$ are chosen in the 2D-like range $\lambda^{-2 / 3}<c / a<1$. The crossover shell $n_{h}$ defines the depth of the layer as $h \simeq 1 / k_{h}$, with $k_{h}=k_{0} \lambda^{n_{h}}$. Denoting with $\left(a_{n}, b_{n}, c_{n}\right)$ the coefficients in $\Phi_{n}(u, u)$ for the generalized shell model (with $a_{n-1}+b_{n}+$ 


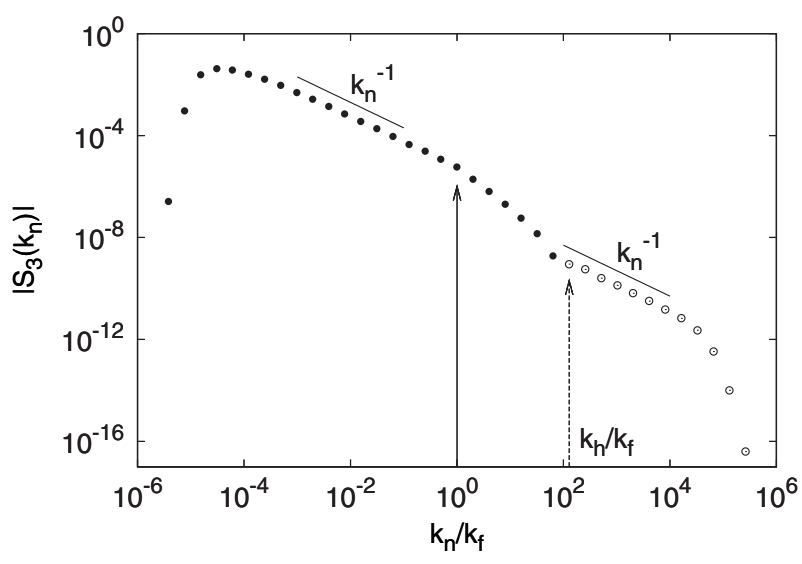

FIG. 1. Absolute value of the third order structure function $\left|S_{3}\left(k_{n}\right)\right|$ (circles). The sign of $S_{3}\left(k_{n}\right)$ is positive (full circles) for $k_{n}<k_{h}$ and negative (empty circles) for $k_{n} \geqslant k_{h}$. Here $k_{h} / k_{f}=2^{7}$.

$c_{n+1}=0$ for energy conservation) we adopt the following choice:

$a_{n}=1, b_{n}=-1-\lambda^{-1 / 2}, c_{n}=\lambda^{-1 / 2}$ for $1 \leqslant n \leqslant n_{h}$,

$a_{n}=1, \quad b_{n}=-1-1 / 2, \quad c_{n}=1 / 2$ for $n_{h} \leqslant n \leqslant N$.

In spite of its simplicity this model is able to reproduce some interesting features of turbulence in thin layers. In particular it has been recently shown that a split energy cascade [12] may be observed if the flow is sustained by an external force with a correlation length larger than the depth of the fluid. A finite fraction of the energy which is injected by the forcing is transferred to lower wave numbers, thus developing an inverse energy cascade. The remnant energy is transferred toward high wave numbers in a direct energy cascade.

In order to investigate such a phenomenon in the shell model we performed numerical simulations of Eq. (1) where the coefficients $(a, b, c)$ are chosen according to Eqs. (3). We allow the shell $n_{h}$, associated to the fluid depth, to vary in the range $n_{f} \leqslant n_{h}<N$. In the simulations the number of shells is $N=40$, and the forcing shell is fixed to $n_{f}=20$. The amplitude of the forcing is chosen in order to provide an energy input $\varepsilon_{I}=1$ and the coefficients of the dissipative term are $\nu=10^{-14}, \mu=10^{2}$, and $p=q=1$. The scale ratio between neighboring shells is $\lambda=2$ and $k_{0}=1 / 2$.

Following [9,11], let us define the third order structure function $S_{3}\left(k_{n}\right)=\operatorname{Im}\left\{\left\langle u_{n-1} u_{n} u_{n+1}^{*}\right\rangle\right\}$, whose sign allows us to discriminate the direction of the energy flux. As shown in Fig. 1, two distinct scaling ranges can be identified. At small wave numbers $k_{n}<k_{f}$ the third order structure function scales as $S_{3}\left(k_{n}\right) \sim k_{n}^{-1}$ and has positive sign, indicating the presence of an inverse cascade of energy with Kolmogorov scaling. The same scaling behavior is recovered also at high wave numbers $k_{n}>k_{h}$, but with a negative sign of $S_{3}\left(k_{n}\right)$, which signals the reversal of the direction of the cascade.

In order to quantify how the energy splits among the two cascades we computed the energy flux defined as $\Pi\left(k_{n}\right)=$ $\sum_{j=n}^{N} \operatorname{Re}\left\{\Phi_{j}(u, u) u_{j}^{*}\right\}$, which shows two plateaus $\Pi\left(k_{n}\right)=$ $-\varepsilon_{\mu}$ for $1 \ll n<n_{f}$ and $\Pi\left(k_{n}\right)=\varepsilon_{v}$ for $n_{f}<n \ll N$ (see Fig. 2). The energy balance in the statistically steady state,

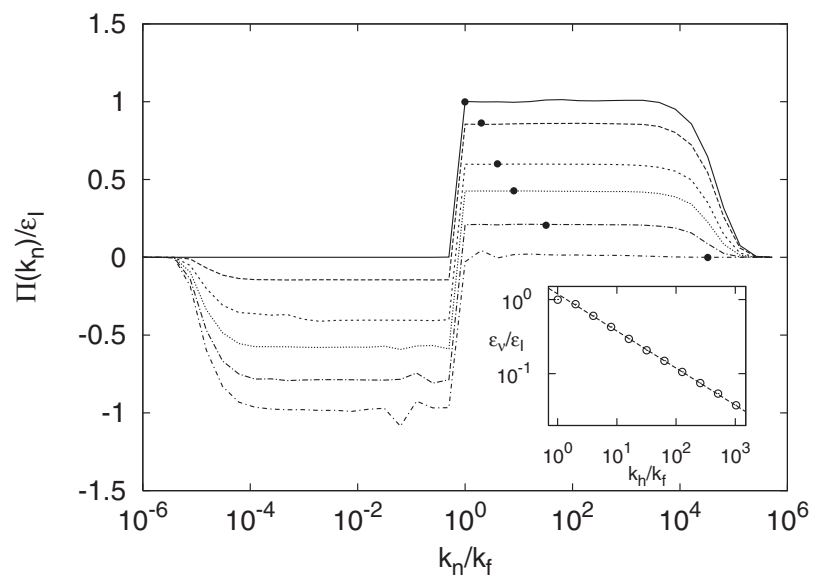

FIG. 2. Energy flux $\Pi\left(k_{n}\right)$ normalized with the input $\varepsilon_{I}$ for increasing values of $k_{h} / k_{f}$ from top to bottom: $k_{h} / k_{f}=$ $2^{0}, 2^{1}, 2^{3}, 2^{5}, 2^{15}$. The shell $k_{h}$ is indicated by black dots on each curve. Inset: Energy flux in the direct energy cascade $\varepsilon_{v}$ as a function of the scale separation $k_{h} / k_{f}$. Dashed line represents the prediction $\varepsilon_{v} / \varepsilon_{I} \sim\left(k_{h} / k_{f}\right)^{-\beta}$.

which is achieved after an initial transient in which the two cascades develop, imposes that the sum of the fluxes in the inverse cascade $\varepsilon_{\mu}$ and direct cascade $\varepsilon_{v}$ equals the energy input $\varepsilon_{I}$. The ratio of the two fluxes depends on the scale separation between the forcing shell $k_{f}$ and the shell associated to the thickness of the fluid layer $k_{h}$. The flux of energy of the inverse cascade reduces as the thickness $h \sim 1 / k_{h}$ increases, and vanishes almost completely when $k_{h} \sim k_{f}$. This is in qualitative agreement with the results of direct numerical simulations [12]. Recent experimental results [1] have shown that an inverse energy cascade can take place also in fluid layers whose depth exceeds the forcing correlation scale. The upscale energy transfer is enhanced by nonlocal interactions due to large coherent structures emerging because of spectral condensation. Of course, this phenomenon is out of reach for shell models, in which only local interactions are included by construction.

An estimate of the amount of energy which is transferred toward high wave numbers can be given by the following argument. In the modified shell model the quantity $H$ is no longer globally conserved by the dynamics. On the other hand it is still locally conserved by the interactions among the shells with $n<n_{h}$, allowing for the development of a direct cascade with constant flux of $H$ in the wave number range $k_{f}<k_{n}<k_{h}$. Such cascade transports also a residual amount of energy $\Pi\left(k_{n}\right) \simeq \varepsilon_{I}\left(k_{n} / k_{f}\right)^{-\beta}$ up to the scale $k_{h}$, where the coefficients $(a, b, c)$ change their values and the 3D-like cascade sets in with a constant flux of energy $\varepsilon_{v} \simeq$ $\varepsilon_{I}\left(k_{h} / k_{f}\right)^{-\beta}$. The fluxes measured in numerical simulations of the model for different values of the scale separation $k_{h} / k_{f}$ are in perfect agreement with this prediction (see inset of Fig. 2).

In analogy with the results obtained for the shell model, it would be tempting to conjecture that in the split cascade process, which takes place in a turbulent layer of depth $h$, the fraction of energy which is transferred toward small viscous scales should be determined by the flux of a partial 


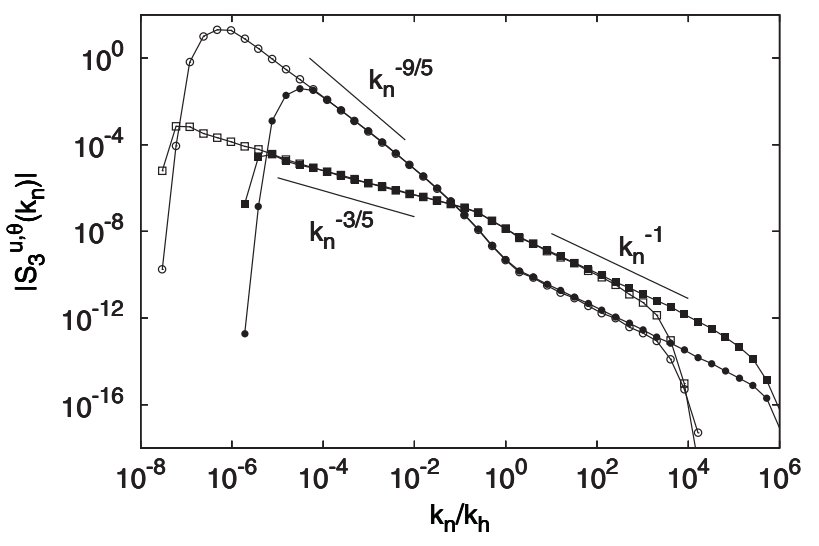

FIG. 3. Absolute value of the third order structure function $\left|S_{3}\left(k_{n}\right)\right|$ for the velocity $u$ (circles), and temperature $\theta$ (squares), for $n_{h}=20$ (black symbols) and $n_{h}=26$ (empty symbols). $S_{3}^{\theta}$ has been multiplied by a factor $10^{-3}$ for plotting purposes.

cascade of enstrophy, which could develop from the forcing scales $\ell_{f}$ down to the scale $h$. It should therefore decay as $\varepsilon_{v} \sim \varepsilon_{I}\left(h / \ell_{f}\right)^{2}$. It would be interesting to test such conjecture with the results of direct numerical simulations.

The geometrical splitting of the energy cascade can be extended to other systems of physical interest. Recently, we have investigated this feature in a Rayleigh-Taylor setup of turbulent convection. In the following we show that also in this case a shell model is able to reproduce the observed phenomenology. We consider a shell model for convective turbulence, in which the turbulent cascade is sustained by an unstable mean temperature gradient $\gamma$ in the vertical direction. Within the Boussinesq approximation, the shell model for the coupled dynamics of variables representing the velocity $u_{n}$ and temperature fluctuations $\theta_{n}$ reads [13]

$$
\begin{gathered}
\frac{d u_{n}}{d t}=i \Phi_{n}(u, u)-D_{n} u_{n}+\beta g \theta_{n}, \\
\frac{d \theta_{n}}{d t}=i \mathcal{L}_{n}(u, \theta)-\kappa k_{n}^{2} u_{n}+\gamma u_{n},
\end{gathered}
$$

where $g$ is the gravity acceleration, $\beta$ is the volume expansion coefficient, and $\kappa$ is the thermal diffusivity. Here, the nonlinear term $\Phi_{n}(u, u)$ and the dissipative term $D_{n} u_{n}$ are chosen as in Eq. (1). The operator $\mathcal{L}_{n}(u, \theta)$, which models the advection term in the equation for the temperature fluctuations, is chosen as $\mathcal{L}_{n}(u, \theta)=k_{m} \theta_{m-1} u_{m}^{*}+k_{m+1} \theta_{m+1} u_{m+1}$.

If the coefficients $(a, b, c)$ of the nonlinear term $\Phi_{n}(u, u)$ are chosen according to Eq. (3), the model provides a suitable tool to investigate the scaling behavior of velocity and temperature fluctuations of a turbulent flow confined in a narrow convective cell, in which one of the two horizontal directions $h$ is much smaller than the other. In general, two different scaling behaviors are expected in turbulent convection. The balance between the buoyancy and inertia forces, which holds for scale larger than the Bolgiano scale $\ell_{B}$, leads to the BolgianoObukhov [14,15] scaling $\delta u(\ell) \simeq \varepsilon_{\theta}^{1 / 5}(\beta g)^{2 / 5} \ell^{3 / 5}$ and $\delta \theta(\ell) \simeq$ $\varepsilon_{\theta}^{2 / 5}(\beta g)^{-1 / 5} \ell^{1 / 5}$, where $\varepsilon_{\theta}$ is flux of the direct cascade of temperature fluctuations. At small scales $\ell \ll \ell_{B}$ the buoyancy force becomes negligible, and the temperature fluctuations are passively transported by the turbulent cascade, leading to the Kolmogorov-Obukhov [16,17] scaling $\delta u(\ell) \simeq \varepsilon^{1 / 3} \ell^{1 / 3}$, $\delta \theta(\ell) \simeq \varepsilon_{\theta}^{1 / 2} \varepsilon^{-1 / 6} \ell^{1 / 3}$. The flux of kinetic energy $\varepsilon$ can be estimated by matching the two scaling regimes at the Bolgiano scale $\varepsilon=\ell_{B}^{4 / 5}(\beta g)^{6 / 5} \varepsilon_{\theta}^{3 / 5}$.

Understanding the dependence of the Bolgiano scale on the control parameters of a real flow is a long standing issue in turbulent convection [18]. Here we use the modified shell model to investigate the dependence of $\ell_{B}$ on the confinement scale $h$. We performed numerical simulations of Eqs. (4) and (5) where the coefficients $(a, b, c)$ are chosen according to Eq. (3), for various values of the shell $n_{h}$, which is associated to the confinement scale $h \sim 1 / k_{h}$. The values of the other parameters used in the simulations are $\beta g=\gamma=1, \kappa=v=$ $10^{-16}, \mu=10^{3} p=1, q=2, \lambda=2$, and $k_{0}=1 / 2$.

As shown in Fig. 3, the third order structure functions, which in the case of the temperature has been defined as $S_{3}^{\theta}\left(k_{n}\right)=\left\langle\left|\theta_{n}\right|^{3}\right\rangle$, clearly show two distinct scaling regimes. At wave numbers $k_{n} \gg k_{h}$ Kolmogorov-Obukhov scaling is observed for temperature and velocity, i.e., $S_{3}^{u, \theta}\left(k_{n}\right) \sim k_{n}^{-1}$. Conversely at small wave numbers $k_{n} \ll k_{h}$ the structure functions follows the Bolgiano-Obukhov scaling $S_{3}^{u}\left(k_{n}\right) \sim$ $k_{n}^{-9 / 5}$ and $S_{3}^{u \theta}\left(k_{n}\right) \sim k_{n}^{-3 / 5}$. The collapse of structure functions obtained for different $n_{h}$ on the scale $k_{h}$ is remarkable and provides compelling evidence that in the shell model considered here the Bolgiano scale is determined by the scale of confinement, i.e., $\ell_{B} \simeq k_{h}^{-1}$. This result confirms our recent findings based on high-resolution direct numerical simulations of turbulent Boussinesq flow confined in a narrow convective cell [19].

It is interesting to observe that the Bolgiano scale is located exactly at the point where the sign of $S_{3}^{u}\left(k_{n}\right)$ changes, indicating the reversal of the energy transfer in the turbulent cascade. In particular the Bolgiano-Obukhov scaling is observed only in the wave-number range where an inverse energy cascade takes place. This is in agreement with previous numerical $[13,20]$ and experimental observations [21,22] which have shown that two-dimensional convective flows are characterized by Bolgiano-Obukhov phenomenology, and corroborates the conjecture that such scaling is associated to an upscale energy transfer.

In conclusion, we have shown that shell models with scaledependent parameters provide a useful tool for investigating the phenomenology of turbulent cascades in fluid layers with large aspect ratio. The proposed model is able to reproduce different phenomena emerging when a $2 \mathrm{D}$ dynamics at large scale coexists with a 3D dynamics at small scales. When mechanically forced Navier-Stokes turbulence is considered, the 2D-3D shell model exhibits a split energy cascade, recently observed in direct numerical simulations and experiments, and allows a detailed study of the dependence of energy partitioning on the thickness of the layer. In the more complex case of turbulence driven by a thermal gradient, the model helps to clarify the natural emergence of the Bolgiano scale from the geometrical confinement of the system. Our results suggest the possibility to apply similar modeling to systems comprising several scale intervals with different dynamical behavior, which are challenging to tackle by means of direct numerical simulation. 
[1] H. Xia, D. Byrne, G. Falkovich and M. Shatz, Nat. Phys. 7, 321 (2011).

[2] M. Shats, D. Byrne, and H. Xia, Phys. Rev. Lett. 105, 264501 (2010).

[3] A. M. Obukhov, Atmos Oceanic Phys. 7, 41 (1971).

[4] E. B. Gledzer, Dokl. Akad. Nauk SSSR 209, 1046 (1973).

[5] M. Yamada and K. Ohkitani, J. Phys. Soc. Jpn. 56, 4210 (1987); Phys. Rev. Lett. 60, 983 (1988).

[6] M. H. Jensen, G. Paladin, and A. Vulpiani, Phys. Rev. A 43, 798 (1991); 45, 7214 (1992).

[7] V. S. L'vov, E. Podivilov, A. Pomyalov, I. Procaccia, and D. Vandembroucq, Phys. Rev. E 58, 1811 (1998).

[8] L. Biferale, Annu. Rev. Fluid Mech. 35, 441 (2003).

[9] T. Gilbert, V. S. L'vov, A. Pomyalov, and I. Procaccia, Phys. Rev. Lett. 89, 074501 (2002).

[10] E. Aurell, G. Boffetta, A. Crisanti, P. Frick, G. Paladin, and A. Vulpiani, Phys. Rev. E 50, 4705 (1994).

[11] P. D. Ditlevsen and I. A. Mogensen, Phys. Rev. E 53, 4785 (1996).
[12] A. Celani, S. Musacchio, and D. Vincenzi, Phys. Rev. Lett. 104, 184506 (2010).

[13] A. Brandenburg, Phys. Rev. Lett. 69, 605 (1992).

[14] R. Bolgiano Jr., J. Geophys. Res. 64, 2226 (1959).

[15] A. Obukhov, Dokl. Akad. Nauk SSSR 125, 1246 (1959).

[16] A. Obukhov, Izv. Aka. Nauk. SSSR: Ser. Geograf. Geofiz 13, 58 (1949).

[17] S. Corrsin, J. Appl. Phys. 22, 469 (1951).

[18] D. Lohse and K. Xia, Annu. Rev. Fluid Mech. 42, 335 (2010).

[19] B. Boffetta, F. De Lillo, A. Mazzino, and M. Musacchio, J. Fluid Mech. (submitted 2011).

[20] A. Celani, A. Mazzino, and L. Vozella, Phys. Rev. Lett. 96, 134504 (2006).

[21] J. Zhang and X. L. Wu, Phys. Rev. Lett. 94, 234501 (2005); J. Zhang, X. L. Wu, and K. Q. Xia, ibid. 94, 174503 (2005).

[22] F. Seychelles, F. Ingremeau, C. Pradere, and H. Kellay, Phys. Rev. Lett. 105, 264502 (2010). 